\title{
Protective Role of Obsessive-Compulsive Symptoms in Schizophrenia
}

\author{
Neda Nasrollahi ${ }^{1 *}$, Imaneh Abasi ${ }^{2}$, Mohammad Reza Mohammadi ${ }^{3}$, Ali Pourramezani ${ }^{4}$ \\ 1. Department of Psychology, Roozbeh Hospital, Tehran University of Medical Sciences, Tehran, Iran. \\ 2. Department of Clinical Psychology, University of Social Welfare and Rehabilitation Sciences, Tehran, Iran. \\ 3. Psychiatry \& Psychology Research Center, Roozbeh Hospital, Tehran University of Medical Sciences, Tehran, Iran. \\ 4. Department of Psychiatry, School of Medicine, Shafa Hospital, Guilan University of Medical Sciences, Rasht, Iran.
}

dteation: Nasrollahi, N., Abasi, I., Mohammadi, M. R., Pourramezani, A. (2016). Protective Role of Obsessive-Compulsive Symptoms in Schizophrenia. Journal of Practice in Clinical Psychology, 4(4), 249-254. https://doi.org/10.18869/acadpub.jpcp.4.4.249

dof : $:$ https://doi.org/10.18869/acadpub.jpcp.4.4.249

\section{Article info:}

Received: 22 Mar. 2016

Accepted: 18 Jum. 2016

\section{Keywords:}

Schizophrenia, Obsessivecompulsive symptoms, Positive symptoms, Negative symptoms

\begin{abstract}
A BSTRACT
Objective: The relationship between obsessive compulsive disorder and schizophrenia has been a matter of dispute for ages. This study aimed to investigate the differences between schizophrenia with obsessive-compulsive symptoms (OCS) and schizophrenia without OCS in positive and negative symptoms of schizophrenia.
\end{abstract}

Methods: Study participants comprised 30 patients with schizophrenia with OCS and 30 patients with schizophrenia without OCS which were enrolled through convenience sampling method from Roozbeh Hospital, Tehran, Iran. Diagnosis was made by a psychiatrist using SCID-I and the data were gathered using positive and negative syndrome scale (PANSS) and Yale-Brown obsessive-compulsive scale (Y-BOC). MANCOVA test was used for analyzing data.

Results: Results indicated that there was not any difference between schizophrenia with OCS group and schizophrenia without OCS group with regard to positive symptoms, but the difference in negative symptoms between two groups was significant.

Conclusion: The present study reveals that OCS may be a protective factor for schizophrenia. Further studies are needed to investigate other factors and mediators involved in this association.

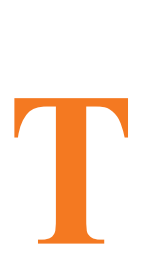

\section{Introduction}

he relationship between obsessive-compulsive disorder and schizophrenia has been a matter of dispute for a long time. The presence of obsessive-compulsive symptoms is rooted in early onset when the first description of illness emerged (Andreasen, 1982). Epidemiological studies in different populations have shown that obsessive-compulsive symptoms (OCS) occur in approximately $8 \%$ to $30 \%$ of patients with schizophrenia
(Tibbo et al., 2000; Byerly et al., 2005; Mohammadi et al., 2007; Tiryaki and Ozkorumak, 2010). This discrepancy in the prevalence of obsessive-compulsive disorder in different societies may be due to different rating scales (Lysaker et al., 2000; Buckley et al., 2009) and or ethnic study groups. In the meantime, there are some similarities in neurological symptoms of OCS and schizophrenia (Harrison et al., 2009).

A growing body of evidence support high prevalence of OCS and obsessive-compulsive disorder (OCD) in pa-

* Corresponding Author:

Neda Nasrollahi, $M A$

Address: Department of Psychology, Roozbeh Hospital, Tehran University of Medical Sciences, Tehran, Iran.

Tel: +98 (21) 55419151

E-mail: n-nasrollahi@farabi.tums.ac.ir 
tients with schizophrenia as well as similar neurological abnormalities in the frontal lobe and basal ganglia with identical changes in neurotransmitter systems, like dopaminergic and serotonergic pathways (Bottas et al., 2005). Hence, many researchers and clinicians have tried to resolve these problems simultaneously and some of them have concluded that the co-occurrence of schizophrenia and OCS may constitute a subtype diagnostic which is called schizo-obssesive disorder (Lysaker et al., 2004).

In line with these findings, some studies have been done to discover the relationships between OCS and clinical manifestations as well as negative and positive symptoms of schizophrenia. The results have been contradictory. Some have reported that the presence of OCS in the schizophrenia worsens the prognosis of disorder and affects the duration of illness and clinical and functional outcomes (Hwang et al., 2000). Some others have shown the positive relationships between delusions and obsessions and also between hallucination and compulsion because of the common mechanism (Guillem et al., 2009). Other studies have found that OCS in schizophrenia correlates with higher levels of both negative and positive symptoms (Lysaker et al., 2002; Nechmad et al., 2003).

Furthermore, Lysaker, Whitney, and Davis in their study found that "participants with significant levels of OCS were significantly and more likely to experience greater levels of hopelessness and endorsed a preference for avoidant focused coping strategies compare to participants without significant OCS" (Lsaker et al., 2006).

Classical psychiatry approach states that the presence of OCS during prodromal period of schizophrenia has a protective effect against some psychotic symptoms (Poyurovsky et al., 1999). Many research findings are consistent with this theory. For example, inverse relationships between somatic obsessions and disorganization and between hoarding/collecting compulsions, and delusions or auditory hallucinations were found in a study (Guillem et al., 2009). Also the results of some other studies revealed that negative symptoms in schizophrenia with OCS are lower than those without such symptoms i.e., lower scores of flat affect subscale from the scale for the assessment of negative symptoms (SANS) in schizoobsessive subgroup (Lysaker et al., 2004; Poyurovsky et al., 1999). At the end, there is also evidence that no significant difference exists between schizophrenia with and without OCS in terms of positive/negative symptoms, clinical manifestations, and prognosis (Byerly et al., 2005; Poyurovsky et al., 2001; Poyurovsky et al., 2006; Faragian et al., 2009; Docherty et al., 2012).
So far the data suggest that OCS in schizophrenia is linked with specified clinical features, but the exact nature of the clinical picture is not clear. Using different research methodologies or differences in the duration of the illness may result in contradicted results. Since there is not enough research to resolve the contradictions, more investigations should be conducted to clarify clinical features of schizophrenia disorder with OCS. Therefore, this study attempts to find answers for the former questions using different methodology and sample.

\section{Methods}

A total of 30 patients with schizophrenia and OCS (70\% male) aged between 22 and 54 years (Mean=34.6; $\mathrm{SD}=8.33 \mathrm{y})$ and 30 patients with schizophrenia without OCS (60\% male) aged between 22 and 65 years (Mean=41.16; $\mathrm{SD}=1.16 \mathrm{y}$ ) were participated in this study. All participants in both groups were matched based on their age, gender, education, marital status, and history of hospitalization.

This study design was cross-sectional. Convenience sampling method was used to recruit the subjects from Roozbeh Psychiatric Hospital. All subjects signed informed consent forms. At first all participants were interviewed by a psychiatrist and met the criteria for schizophrenia based on DSM-IV-TR, then the diagnosis was confirmed by a clinical psychologist using structured clinical interview for DSM-IV-TR Axis I Disorders (SCID-I). The participants were interviewed face-toface by a trained researcher. The presence of OCS was checked by at least two expert psychiatrists. Samples had different types of schizophrenia and aged 18 to 65 years.

Outpatient participants were stable and their prescribed medications have not changed within the last year. Inpatient participants who were cooperative enough underwent an interview as well. Exclusion criteria were as follows: 1) having mental retardation; 2) existence of extrapyramidal symptoms 3 ) past or current drug abuse or dependence; and 4) having comorbid mood disorders. All participants were assessed and interviewed individually in a quiet room. A clinical psychologist assessed the severity of OCS by using the Yale-Brown obsessivecompulsive scale (Y-BOC). For diagnosing the schizophrenia group with OCS, a minimum score of 8 in YBOCS and minimum of 6 months duration of symptoms were required. Also positive and negative symptoms of schizophrenia were rated by a clinical psychologist using positive and negative syndrome scale (PANSS). 
In order to maximize the accuracy of assessments, families of clients were also interviewed. The interviews were done by researcher after informed consent forms were obtained. Participants were told that their demographic information would be kept confidential.

The Structured Clinical Interview for Diagnostic and statistical manual of mental disorders, fourth edition axis I disorders (SCID-I) (Spitzer and Gibbon, 1997) is a semi-structured and reliable instrument for the measurement and diagnosis of selected Axis I mental syndromes and disorders according to the criteria of the Diagnostic and Statistical Manual. Its inter-rater reliability (к) is $0.70-1$. The Persian version of SCID-I (Sharifi et al., 2009) has been reported to have good reliability $(\kappa=0.52$ for current diagnosis and 0.55 for lifetime diagnosis).

The checklist of Yale-Brown Obsessive-Compulsive scale (Y-BOC) is designed to rate the severity and type of symptoms in patients with OCD. It is a part of overall Y-BOCS scale which was developed by Goodman and his colleagues (Goodman et al., 1989). In this checklist, the scores of $0,1,2,3$, and 4 were assigned to lack of problems, mild, moderate, severe, and very severe, respectively. Typically, a total score is reported based on the sum of all items. This scale has acceptable reliability and validity. Internal consistency coefficient ( $\alpha$ coefficient) has been reported to be $89 \%$ (Kim et al., 1989). The Iranian version of this scale has acceptable reliability, too. In a study on 50 Iranian patients, test-retest reliability (two weeks interval) was 84\% (Institute, 1994).

The Positive and Negative Syndromes Scale (PANSS) consists of 30 items with three subscales; negative, positive, and general psychopathology for schizophrenia. Internal reliability $(\alpha)$ for positive, negative, and general psychopathology has been reported to be $0.73,0.83$, and 0.79 , respectively. Test-retest reliability across 3 to 6 months for inpatient phase has shown to be $0.80,0.68$, and 0.60 for positive, negative, and general psychopathology, respectively. Consistency among dependent raters were also acceptable and calculated as $0.83,0.85$, and 0.87 re- spectively for the mentioned subscales (Kay et al., 1988). This scale has been reported to have acceptable validity in Iranian population, too (Forouzandeh et al., 2009).

To study differences between schizophrenia with OCS sample and schizophrenia without OCS sample in terms of positive and negative symptoms of schizophrenia, multivariate analysis of covariance (MANCOVA) were performed. Differences between two groups with regard to the background variables (age, gender, marital status, number of hospitalizations, and duration of hospitalization) were assessed by using Chi-square and Independent sample t tests. There were no differences in background variables between two groups except for the duration of hospitalization and age. So these variables were entered as covariates. When MANOVA yielded a significant main effect, multiple pair-wise comparisons with Bonferroni correction were used then.

\section{Results}

This study aimed to investigate the differences between schizophrenia with OCS and schizophrenia without OCS with regard to positive and negative symptoms of schizophrenia. MANCOVA showed an overall multivariate differences in positive and negative symptoms of schizophrenia between schizophrenia with OCS and schizophrenia without OCS (Wilks Lambda $=0.794 ; \mathrm{F}(1,53)=6.501$; $\mathrm{P}<0.003$ ). In other words, there were significant differences between two groups with regard to positive and negative symptoms of schizophrenia. Subsequent post hoc tests showed significant differences between two groups with regard to negative symptoms, but no significant difference with regard to positive symptoms. Results of MONCOVA and Bonferroni post hoc test are presented in Table 1.

\section{Discussion}

The current study was conducted to compare schizophrenic patients plus OCS with schizophrenic patients without OCS with regard to positive and negative symptoms. Findings revealed that there was a significant difference between two groups regarding the negative

Table 1. Means, standard deviations and results of MANCOVA of dependent variables.

\begin{tabular}{|c|c|c|c|c|c|c|c|c|}
\hline \multirow{2}{*}{ Variables } & \multicolumn{2}{|c|}{ Schizophrenia With OCS } & \multicolumn{2}{|c|}{ Schizophrenia Without OCS } & \multirow{2}{*}{$\mathbf{F}$} & \multirow{2}{*}{ Effect Size } & \multirow{2}{*}{ Sig. } & \multirow{2}{*}{ I-J } \\
\hline & Mean & SD & Mean & SD & & & & \\
\hline $\begin{array}{l}\text { Negative } \\
\text { symptoms }\end{array}$ & 21.46 & 7.78 & 29.93 & 6.38 & 13.19 & 0.2 & $0.001+$ & $-7.43^{*}$ \\
\hline $\begin{array}{l}\text { Positive } \\
\text { symptoms }\end{array}$ & 23.5 & 8.1 & 25.8 & 6.2 & 0.23 & 0.05 & 0.63 & \\
\hline
\end{tabular}

I: Schizophrenia with OCD symptoms; J: Schizophrenia without OCD symptoms.

†: Significant difference using the Bonferroni post hoc test. 
symptoms of schizophrenia. But the differences between two study groups were not significant with regard to positive symptoms. Contradictions among studies on the relationship between OCS or OCD and the severity of schizophrenia symptoms are not disputable. Findings of the present study are consistent with some previous study results and opposed to others.

The results of this study showed that there was a difference between schizophrenia with OCS and schizophrenia without it with regard to negative symptoms so that schizophrenia group with OCS reported less negative symptoms than those without OCS. These findings revive the belief that the presence of obsessive-compulsive symptoms in schizophrenia may act as a protecting factor and indicates good prognosis (Stengel, 1945).

Result of the present research is in line with some prior studies, which showed that patients with schizophrenia and OCS present fewer negative symptoms (Lysaker et al., 2004; Poyurovsky et al., 1999). Lysaker reported that OCS is associated with fewer negative symptoms in patients with schizophrenia (Lysaker et al., 2004). Nasrollahi et al. (2012) also conducted some research on the relationship between positive and negative symptoms of schizophrenia and OCS. Their results indicated that there was a negative relationship between obsession and negative symptoms of schizophrenia, but their study lacked a control group. The present study supports previous results with adding a control group. Above studies reveal that schizophrenia may have a specific subtype named schizo-obsessive subtype and OCS may have a protective effect on schizophrenia symptoms.

Yet, there are some other findings that oppose the present study; they reported that subjects with schizophrenia and OCS present more negative symptoms (Owashi et al., 2010; Seong Koo et al., 2008). In another study on hospitalized schizophrenia, no significant relationship was observed between OCS and negative and positive symptoms of schizophrenia (Poyurovsky et al., 2001). Ozay Ozdemir et al. (2003) studied the clinical features of OCD in patients with schizophrenia and compared them with patients without schizophrenia. They found that the severity, characteristics, and frequency of OCS in patients with schizophrenia are similar to those of patients without schizophrenia.

There are some ways to reconcile these discrepancies. One of them may owe to different samples and methodologies employed, or differences in the duration of the illness the patients had. Such lack of harmony in findings may also be due to several other factors. One of the most important factors is different definitions of OCS. When a dimensional definition is used OCS-schizophrenia showed a greater severity of psychotic symptoms than non-OCS schizophrenia (Cunill et al., 2009). Moreover, the heterogeneity among methods of analyzing data and diversity in samples can lead to different results. Differences in inclusion of inpatient and outpatient may be another source of heterogeneity (Cunill et al., 2009). Furthermore, Lieuwe de Haan et al. (2013) reported the role of quality of life and well-being in the presence of OCS in patients with first episode of schizophrenia. This may explain at least some differences in different societies.

The most important limitations for this study are its cross-sectional design and using multivariate method. Furthermore, since the information obtained from participants was retrospective there might be the risk of forgetting and biasing that should be considered. Thus, further investigations with different methods and designs are needed to confirm the achieved results.

In conclusion, the current study showed the protective effect of OCS in schizophrenia disorder. In other words, the presence of OCS in patients with schizophrenia disorder reduce the negative symptoms which could result in the maintaining psychological functions of the patients. Furthermore, the finding revealed that the cooccurrence of schizophrenia with OCS was related to a different clinical picture which was a specific subtype of schizophrenia under the title of schizo-obsessive subtype. The relationship between OCS and positive/negative symptoms of schizophrenia has been a subject of debate for many years and still is.

This relation is nonlinear and there may be different mechanisms and mediators explaining different pathways, so further research are needed to illuminate these pathways.

\section{Acknowledgements}

We appreciate Department of Psychology, also Psychiatry and Psychology Research Center of Roozbeh Hospital affiliated to Tehran University of Medical Sciences for their kind help in this research. The current research hasn't received any financial support.

\section{Conflict of Interest}

The authors declared no conflict of interests. 


\section{References}

Andreasen, N. C. (1982). Negative symptoms in schizophrenia: Definition and reliability. Archives of General Psychiatry, 39(7), 784-88. doi: 10.1001/archpsyc.1982.04290070020005

Bottas, A., Cooke, R. G., \& Richter, M. A. (2005). Comorbidity and pathophysiology of obsessive-compulsive disorder in schizophrenia: is there evidence for a schizo-obsessive subtype of schizophrenia? Journal of Psychiatry \& Neuroscience, 30(3), 187. PMID: 15944743

Buckley, P. F., Miller, B. J., Lehrer, D. S., \& Castle, D. J. (2009). Psychiatric comorbidities and schizophrenia. Schizophrenia Bulletin, 35(2), 383-402. doi: 10.1093/schbul/sbn135

Byerly, M., Goodman, W., Acholonu, W., Bugno, R., \& Rush, A. J. (2005). Obsessive compulsive symptoms in schizophrenia: frequency and clinical features. Schizophrenia Research, 76(2), 309-16. doi: 10.1016/j.schres.2005.02.004

Cunill, R., Castells, X., \& Simeon, D. (2009). Relationships between obsessive-compulsive symptomatology and severity of psychosis in schizophrenia: A systematic review and meta-analysis. Journal of Clinical Psychiatry, 70(1), 70-82. doi: 10.4088/jcp.07r03618

Docherty, A. R., Coleman, M. J., Tu, X., Deutsch, C. K., Mendell, N. R., \& Levy, D. L. (2012). Comparison of putative intermediate phenotypes in schizophrenia patients with and without obsessive-compulsive disorder: Examining evidence for the schizo-obsessive subtype. Schizophrenia Research, 140(1), 8386. doi: 10.1016/j.schres.2012.06.033

Faragian, S., Pashinian, A., Fuchs, C., \& Poyurovsky, M. (2009) Obsessive-compulsive symptom dimensions in schizophrenia patients with comorbid obsessive-compulsive disorder. Progress in Neuro-Psychopharmacology \& Biological Psychiatry, 33(6), 1009-12. doi: 10.1016/j.pnpbp.2009.05.008

Foruzandeh, N., Foruzandeh, M., Delaram, M., Darakhshandeh, S., Deris, F., Hasanpour Dehkordi A., et al. [The effect of occupational therapy on some aspects of quality of life in schizophrenic patients (Persian)]. Journal of Shahrekord University of Medical Sciences, 10(4), 51-57.

Goodman, W. K., Price, L. H., Rasmussen, S. A., Mazure, C., Fleischmann, R. L., Hill, C. L., et al. (1989). The Yale-Brown obsessive compulsive scale: I. Development, use, and reliability. Archives of General Psychiatry, 46(11), 1006-11. doi: 10.1001/ archpsyc.1989.01810110048007

Guillem, F., Satterthwaite, J., Pampoulova, T., \& Stip, E. (2009). Relationship between psychotic and obsessive compulsive symptoms in schizophrenia. Schizophrenia Research, 115(2), 358-62. doi: 10.1016/j.schres.2009.06.004

de Haan, L., Sterk, B., \& Valk, R. (2013). Presence of obsessive compulsive symptoms in first-episode schizophrenia or related disorders is associated with subjective well-being and quality of life. Early Intervention in Psychiatry, 7(3), 285-90. doi: 10.1111/j.1751-7893.2012.00377.x

Harrison, B. J., Soriano-Mas, C., Pujol, J., Ortiz, H., López-Solà, M., Hernández-Ribas, R., et al. (2009). Altered corticostriatal functional connectivity in obsessive-compulsive disorder. Archives of General Psychiatry, 66(11), 1189-200. doi: 10.1001/ archgenpsychiatry.2009.152

Hwang, M. Y., Morgan, J. E., \& Losconzcy, M. F. (2000). Clinical and neuropsychological profiles of obsessive-compulsive schizophrenia: A pilot study. Journal of Neuropsychiatry and Clinical Neurosciences, 12(1), 91-94. doi: 10.1176/jnp.12.1.91

Kaplan, H., \& Kaplan, S. B. (2007). Kaplan \& Sadock's synopsis of psychiatry: Behavioral sciences/clinical psychiatry [N. Pour Afkari (Persian trans.)]. Tehran: Shahr-e Ab.

Kay, S. R., Opler, L. A., \& Lindenmayer, J. P. (1988). Reliability and validity of the positive and negative syndrome scale for schizophrenics. Psychiatry Research, 23(1), 99-110. doi: 10.1016/0165-1781(88)90038-8

Kim, S. W., Dysken, M. W., \& Katz, R. (1989). Rating scales for obsessive compulsive disorder. Psychiatric Annals, 19(2), 7479. doi: 10.3928/0048-5713-19890201-07

Koo, M. S., Koo, M. S., Kim, C. H., Suh, H. S., \& Lee, H. S. (2008) Clinical characteristics of obsessive compulsive disorder with schizophrenia. Schizophrenia Research, 102(1), 227-28. doi: 10.1016/s0920-9964(08)70686-8

Lysaker, P. H., Bryson, G. J., Marks, K. A., Greig, T. C., \& Bell, M. D. (2002). Association of obsessions and compulsions in schizophrenia with neurocognition and negative symptoms. Journal of Neuropsychiatry \& Clinical Neurosciences, 14(4), 44953. doi: $10.1176 /$ jnp.14.4.449

Lysaker, P. H., Lancaster, R. S., Nees, M. A., \& Davis, L. W. (2004). Patterns of obsessive-compulsive symptoms and social function in schizophrenia. Psychiatry Research, 125(2), 13946. doi: 10.1016/j.psychres.2003.12.007

Lysaker, P. H., Marks, K. A., Picone, J. B., Rollins, A. L., Fastenau, P. S., \& Bond, G. R. (2000). Obsessive and compulsive symptoms in schizophrenia: clinical and neurocognitive correlates. Journal of Nervous \& Mental Disease, 188(2), 78-83. doi: 10.1097/00005053-200002000-00003

Lysaker, P. H., Whitney, K. A., \& Davis, L. W. (2006). Obsessivecompulsive and negative symptoms in schizophrenia: Associations with coping preference and hope. Psychiatry Research, 141(3), 253-59. doi: 10.1016/j.psychres.2005.09.016

Mohammadi, M. R., Ghanizadeh, A., \& Moini, R. (2007). Lifetime comorbidity of obsessive-compulsive disorder with psychiatric disorders in a community sample. Depression $\mathcal{E}$ Anxiety, 24(8), 602-07. doi: 10.1002/da.20259

Nasrollahi, N., Bigdelli, I., Mohammadi, M. R., \& Makvand Hosseini, Sh. (2012). The relationship between obsessions and compulsions and negative and positive symptoms in schizophrenia. Iranian Journal of Psychiatry, 7(3), 140-45. PMCID: PMC3488870

Nechmad, A., Ratzoni, G., Poyurovsky, M., Meged, S., Avidan, G., Fuchs, C., et al. (2003). Obsessive-compulsive disorder in adolescent schizophrenia patients. American Journal of Psychiatry, 160(5), 1002-004. doi: 10.1176/appi.ajp.160.5.1002

Owashi, T., Ota, A., Otsubo, T., Susa, Y., \& Kamijima, K. (2010). Obsessive-compulsive disorder and obsessive-compulsive symptoms in Japanese in patients with chronic schizophrenia-a possible schizophrenic subtype. Psychiatry Research, 179(3), 241-46. doi: 10.1016/j.psychres.2009.08.003

Ozdemir, O., Tukel, R., Turksoy, N., \& Ucok, A. (2003). Clinical characteristics in obsessive-compulsive disorder with schizophrenia. Comprehensive Psychiatry, 44(4), 311-16. doi: 10.1016/ S0010-440X(03)00093-2 
Poyurovsky, M., Bergman, J., \& Weizman, R. (2006). Obsessive-compulsive disorder in elderly schizophrenia patients. Journal of Psychiatric Research, 40(3), 189-91. doi: 10.1016/j. jpsychires.2005.03.009

Poyurovsky, M., Fuchs, C., \& Weizman, A. (1999). Obsessivecompulsive disorder in patients with first-episode schizophrenia. American Journal of Psychiatry, 156(12), 1998-2000. doi: 10.1176/ajp.156.12.1998

Poyurovsky, M., Hramenkov, S., Isakov, V., Rauchverger, B., Modai, I., Schneidman, M., et al. (2001). Obsessive-compulsive disorder in hospitalized patients with chronic schizophrenia. Psychiatry Research, 102(1), 49-57. doi: 10.1016/s01651781(01)00238-4

Sharifi, V., Assadi, S. M., Mohammadi, M. R., Amini, H., Kaviani, H., Semnani, Y., et al. (2009). A Persian translation of the structured clinical interview for diagnostic and statistical manual of mental disorders: Psychometric properties. Comprehensive Psychiatry, 50(1), 86-91. doi: 10.1016/j.comppsych.2008.04.004

Spitzer, R. L., Gibbon, M. (1997). User's guide for the structured clinical interview for Dsm-IV Axis I disorders: Scid-1 clinician version. Arlington, V. A.: American Psychiatric Publication.

Stengel, E. (1945). A study on some clinical aspects of the relationship between obsessional neurosis and psychotic reaction types. British Journal of Psychiatry, 91(383), 166-87. doi: 10.1192/bjp.91.383.166

Tibbo, P., Kroetsch, M., Chue, P., \& Warneke, L. (2000). Obsessive-compulsive disorder in schizophrenia. Journal of Psychiatric Research, 34(2), 139-46. doi: 10.1016/ s0022-3956(99)00048-

Tiryaki, A., \& Özkorumak, E. (2010). Do the obsessive-compulsive symptoms have an effect in schizophrenia? Comprehensive Psychiatry, 51(4),357-62. doi:10.1016/j.comppsych.2009.10.007 